\title{
Preface to the Series on Electrochemistry
}

The scope of electrochemistry having broadened tremendously within the last ten years has become a remarkably diverse science. In the field of electroorganic synthesis, for example, selectivity has been improved by use of electrogenerated reagents, energy uptake lowered and space-time yields have been improved by using mediated reactions. In addition, electroorganic chemistry has been efficiently applied to the synthesis of key building blocks for complex molecules and has established its role as a new tool in organic synthesis. However electrochemistry has also found new and interesting applications in quite different fields of chemistry. Photoelectrochemistry, as one example, is not only valuable for transformations of organic molecules but also for the very important goal of energy conversion. More insight has been gained in the processes occurring on illuminated semiconductor electrodes and micro particles. Designing the composition of electrode surfaces can lead to the selective activation of electrodes. Electrochemical sensors and techniques present new opportunities for the analysis of biological compounds in medicine and biology. Research in the field of conducting polymers is very intensive because of interesting potential applications.

Therefore I am very happy that Springer-Verlag has decided to account for these important developments by introducing a series of volumes on new trends in electrochemistry within its series Topics in Current Chemistry. The volumes will cover the important trends in electrochemistry as outlined above in the following manner:

Electroorganic Synthesis by Indirect Electrochemical Methods; New Applications of Electrochemical Techniques;

Recent Development in Electroorganic Synthesis.

The guest editor is very happy and thankful that well-known experts who are actively engaged in research in these fields have agreed to contribute to the volumes. It is hoped that this collection of reviews is not only valuable to investigators in the respective fields but also to many chemists who are not so familiar with electrochemistry. 
